\title{
Evaluating ambulatory care training in Firoozgar hospital based on Iranian national standards of undergraduate medical education
}

\author{
Foroogh Sabzghabaei ${ }^{1}$, Mahla Salajeghe ${ }^{2 *}$, Seyed Kamran Soltani Arabshahi ${ }^{3}$ \\ Received: 10 Nov 2016 \\ Published: 17 Dec 2017
}

\begin{abstract}
Background: In this study, ambulatory care training in Firoozgar hospital was evaluated based on Iranian national standards of undergraduate medical education related to ambulatory education using Baldrige Excellence Model. Moreover, some suggestions were offered to promote education quality in the current condition of ambulatory education in Firoozgar hospital and national standards using the gap analysis method.

Methods: This descriptive analytic study was a kind of evaluation research performed using the standard check lists published by the office of undergraduate medical education council. Data were collected through surveying documents, interviewing, and observing the processes based on the Baldrige Excellence Model. After confirming the validity and reliability of the check lists, we evaluated the establishment level of the national standards of undergraduate medical education in the clinics of this hospital in the 4 following domains: educational program, evaluation, training and research resources, and faculty members. Data were analyzed according to the national standards of undergraduate medical education related to ambulatory education and the Baldrige table for scoring. Finally, the quality level of the current condition was determined as very appropriate, appropriate, medium, weak, and very weak.

Results: In domains of educational program $62 \%$, in evaluation $48 \%$, in training and research resources $46 \%$, in faculty members $68 \%$, and in overall ratio, $56 \%$ of the standards were appropriate.

Conclusion: The most successful domains were educational program and faculty members, but evaluation and training and research resources domains had a medium performance. Some domains and indicators were determined as weak and their quality needed to be improved, so it is suggested to provide the necessary facilities and improvements by attending to the quality level of the national standards of ambulatory education.
\end{abstract}

Keywords: Ambulatory care training, Program evaluation, Iranian national standards of undergraduate medical education

Copyright $₫$ Iran University of Medical Sciences

Cite this article as: Sabzghabaei F, Salajeghe M, Soltani Arabshahi SK. Evaluating ambulatory care training in Firoozgar hospital based on Iranian national standards of undergraduate medical education. Med J Islam Repub Iran. 2017 (17 Dec);31:99. https://doi.org/10.14196/mjiri.31.99

\section{Introduction}

Iranian medical universities need to improve the quality of their medical education and constantly evaluate its status because of their important role in promoting medical education and providing health care and sanitation services.

Clinical training mainly concerns training and learning about the patients and their problems, which is the backbone of medical education. Since most of the patients seek

Corresponding author: Mahla Salajeghe, mahla.salajegheh90@gmail.com

1. Department of Internal Medicine, Iran University of Medical Sciences, Tehran, Iran. 2. Education Development Center, Kerman University of Medical Science, Kerman, Iran. 3. Department of Medical Education, Faculty of Medicine, Iran University of Medical Sciences, Tehran, Iran. ambulatory care services in ambulatory care centers and only a few of them go to specialty hospitals, it is clear that training the medical students in wards only fails to familiarize them with health care and treatment problems of the society $(1,2)$. According to the rules set by The Ministry of Health and Medical Education (MHME), 50\% of the training for the medical students should be in ambulatory care

$\uparrow$ What is "already known" in this topic:

Evaluating the quality of education is one of concerns in Iranian medical education, specifically in ambulatory section. That highlights the urgency of paying extra attention to the effectiveness of the higher education system.

$\rightarrow$ What this article adds:

The national standards of ambulatory training in undergraduate medical education are highly useful in evaluating ambulatory training. There is an appropriate approach towards applying the national standards of ambulatory training in undergraduate medical education in Firoozgar Hospital. 
centers (3). Specific features of medical training in ambulatory centers on the one hand and the fact that the main portion of the general practitioners' activities is performed in ambulatory care centers on the other hand have made it impossible for traditional methods of education to be satisfactory even with massive modifications done to them. Despite their plentiful advantages, reaching efficiency in clinical training in ambulatory care unit is challenging (4). However, no comprehensive study has been conducted in Iran to cover different aspects of the quality of ambulatory care training. However, some studies such as Alizadeh et al. in Shiraz indicated that most of the learners believed the present ambulatory care training falls short of meeting the forthcoming needs and requirements of general practitioners (5). Payvandi et al. in Semnan (6) and Amini et al. in Tabriz (7) found that more than half of the professors considered the ambulatory care training to be insufficient; moreover, the unsatisfactory findings of studies done by Shaigan et al. in Isfahan University of Medical Sciences and Payvandi et al. in Semnan University of Medical Sciences about ambulatory care training $(8,9)$ confirmed the insufficiency and the shortcomings of this significant part of medical education in Iran. Such findings indicate the lack of sufficient attention to ambulatory training nationwide and the absence of a proper and binding plan to improve the quality of ambulatory trainings.

On the other hand, analyzing the trends in higher education in Iran reveals that the utmost concern in Iranian educational issues in the recent years has rested upon the declining trends in the indicators of the quality of education, which highlights the urgency of paying extra attention to the effectiveness of the higher education system (10). Also, it should be taken into account that evaluating and improving the quality of education is one of the important objectives in higher education worldwide (11).

According to the Fifth Plan of Development and the ratifications of Planning Council of MHME, to implement the general policies of the country, special heed was cast upon inaugurating and streamlining the accreditation and evaluation systems of the institutions and educational courses (12). Henceforth, considering the society's ethical codes of conduct and its extraordinary conditions concerning education and health care services, the medical universities of Iran are to constantly observe and check the status quo via premeditated and prearranged criteria.

All these factors stress the need for an appropriate and practical evaluating system in medical universities, specifically in ambulatory section considering its conditions, characteristics, and special whereabouts to evaluate the status quo and improve the quality of education (13).

To tackle the paramount issue of evaluating the quality of education in universities, various mechanisms and patterns are being deployed; and in the meanwhile, accreditation as a well-known apparatus is being taken advantage of in top universities and has a substantial place in evaluating and monitoring stages (14). Accreditation is granting a license to or certifying an educational center, which is being judged by the authorities in a certain field and has observed all specifications (15). By ambulatory training accreditation we mean that considering all the necessary standards for each constituent element of the program and comparing them with those elements in ambulatory centers, we should evaluate the aforementioned elements to eliminate all the deficiencies and faults and meet the acceptable international criteria in quality for ambulatory centers (16).

One of the most creditable guidelines for accreditation of medical education is that of World Federation of Medical Education (WFME), with which different countries have evaluated their medical university education systems (1723).

The systematized measures to accredit undergraduate medical education in Iran commenced in $3 / 3 / 2007$, with preparing Iranian national standards of undergraduate medical education in 4/16/2007, which were communicated to the medical universities to take effect to improve the quality of medical education $(24,25)$.

Therefore, with regard to the obligation for deploying this plan and the need in Firoozgar hospital for evaluating and improving the quality of its ambulatory training, a team consisting of ambulatory care professors of this hospital conjoined with medical education experts in coordination with the national and worldwide movement to evaluate and improve the quality of education in ambulatory centers, instigated the necessary measures to conduct this study.

On the other hand, deploying standards is a management activity, which is closely interconnected with organizational excellence models. Malcolm Baldrige Excellence Model, which has optimized total quality management (TQM) techniques for education-based health care organizations, is able to create a mutual understanding and conceptual common ground among professors, medical education development centers, and the managers. In this study, to elucidate the management-related obstacles confronting the deployment of the national standards of undergraduate medical education in ambulatory training section, Baldrige framework's scoring matrix was used. Using Baldrige framework's scoring matrix, one can evaluate the deployment of a criterion along 4 dimensions:

Approach: In this dimension, the educational plan (process) of the managers and their attitude towards the demand of deployment of such a criterion is evaluated.

Deployment: In this dimension, the measures taken towards deploying the national standards of ambulatory training throughout the organization or each section and their indicators are evaluated.

Learning: In this dimension, the data collected following the deployment in each section of the standard and its indicators would be systematically assessed and the feedback is offered to the people in charge to improve the educational plan. This will lead to the realization of organizational learning and constant improvement of the educational plan.

Integration: In this dimension, data collected from relevant indicators in one section or other sections of the standard system would be comprehensively and integrally studied and interpreted improvement-wise.

The present study aimed at determining the degree of the conformity of ambulatory training status quo in Firoozgar hospital with Iranian national standards of undergraduate medical education related to ambulatory training based on Baldrige Excellence Model. 


\section{Methods}

This was a descriptive analytic research. Surveying the documents, interviewing, and field observation of ambulatory training processes, with the help of experts of each related specialized field and according to sections and indexes of the national standards of undergraduate medical education for ambulatory training were the techniques adopted in this study to evaluate the status of ambulatory training in Firoozgar hospital. Furthermore, a Ph.D. student of medical education held a number of training sessions for the experts, who were specialists in various but relevant fields in educational ambulatory centers. In these sessions, it was reviewed how to fill out and score index checklists using Baldrige Education Scoring Matrix and their [specialists'] questions were answered and discussed. Through triangulation of data collection methods-interviewing, observation, and surveying the documents - and also triangulation of the sources from which we collected the dataprofessors, students, and education managers - we tried to increase the reliability and verifiability of the data. The research tool used was the standard checklists released from the secretariat of undergraduate medical education council, which evaluated the 4 domains of educational program, evaluation, training and research resources, and faculty members in the clinics of Firoozgar hospital. Among the indexes of the 7 checklists, which covered the whole course of medical education, the indexes of 4 checklists related to ambulatory training were selected using expert panel method to ensure the face and content validity. To ensure the reliability of the checklists, we used interrater reliability, which was tested using kappa coefficient.

The education program checklist included 4 subcategories (the development and communication of educational goals, training and the duties of the teachers and students, outcomes of educational program, content of the curriculum, and educational strategies), 9 indexes, and 23 indicators.

The evaluation checklists included 1 subcategory (program evaluation), 4 indexes, and 15 indicators.

The training and research resources checklist included 2 subcategories (space and facilities, and development of medical education), 3 indexes, and 7 indicators.

The faculty members' checklist included 1 subcategory (recruitment of faculty), 3 indexes, and 3 indicators.

To calculate the sample size, since the main body of the education was provided in major clinics, those clinics were selected as samples through census method. Major specialties in this hospital are 3 main groups of internal medicine, surgery, and gynecology, which are practiced in 16 clinics.

The appropriateness of the current condition of ambulatory training in Firoozgar hospital in each national standard indicator, based on the practical definition of the study, was rated as follows: 1: very appropriate (gaining more than $80 \%$ of the score); 2: appropriate (gaining more than $60 \%$ up to $80 \%$ of the score); 3 : medium (gaining more than $40 \%$ up to $60 \%$ of the score); 4 : weak (gaining more than $20 \%$ up to $40 \%$ of the score); 5 : very weak (gaining less than $20 \%$ of the score).

For data analysis, the national standards of undergraduate medical education (NSUME) in ambulatory training and
Baldrige Scoring Matrix were used and gap analysis was utilized to determine the gap between status quo and NSUME.

Scoring was based on the scale of 0 to 100 for answering each question on the checklists, based on Baldrige Excellence Model Scoring Matrix, with score coefficient of 1 for approach, score coefficient of 2 for deployment, 3 for learning, and 4 for integration. Therefore, each indicator in each of the 3 techniques of data collection could receive between 0 to 1000 score, and the average score of the 3 indicators would be considered that indicator's score. Likewise, the average score of all indicators in each domain would be considered the score and the percentage of the gained score for it. Using this matrix, the level of all domains separately and all highlighted clinics of Firoozgar hospital were determined in 4 dimensions of approach, deployment, learning, and integration.

\section{Results}

The 16 studied clinics were divided into 3 groups of internal medicine, surgery, and gynecology. In surgery group, there were 5 clinics of general surgery ( 2 clinics), neurosurgery, orthopedics, and ENT (ear, nose, and throat). In internal medicine group, there were 9 clinics of hematology, neurology, rheumatology, cardiology, pulmonary, infectious diseases, gastroenterology, and nephrology ( 2 clinics), and 2 clinics were evaluated in gynecology group.

\section{The domain of education programs (EP)}

Based on the evaluation results of the clinics in the surgery group, it was revealed that this group's performance in the education program (EP) domain was appropriate (Table 1). This achievement was due to high scores in the following indexes: preparing and communicating objectives and duties (PCOD), curriculum of science stage (CSS), curriculum of clinical stage (CCS), applying the latest educational strategies (ALES), and professors and assistants' cooperation (PAC). In this domain, the performance in other indexes was evaluated as medium.

The internal medicine group had also an appropriate performance in this domain (Table 1). The achievement was mainly due to high scores in the following indexes: PCOD, CCS, ALES, and PAC. In this domain other indexes scored medium.

The gynecology group showed an appropriate performance in EP domain (Table 1). This achievement was mainly due to high score in the following indexes: PCOD, $\mathrm{CSS}, \mathrm{CCS}, \mathrm{PAC}$, and observing professional values and requirements (OPVR). In this domain other indexes scored medium.

\section{The domain of evaluation}

The results revealed that the performance of the surgery group clinics in this domain scored medium (Table 2). In this domain, preparing and announcing evaluation procedure (PAEP) index was the most appropriate. On the contrary, test validation (TV) and collaboration in assessment (CA) indexes scored very weak and index of commensurability of evaluation with educational objectives (CEEO) scored medium. The main weakness in this index was the 
Table 1. The status of the education program domain based on national standards of ambulatory training in Firoozgar hospital using Baldrige Excellence Model Matrix

\begin{tabular}{|c|c|c|c|c|}
\hline & $\begin{array}{l}\text { Total score } \\
\text { Triangulation and weighting }\end{array}$ & $\begin{array}{l}\text { Clinics of internal medi- } \\
\text { cine group }\end{array}$ & $\begin{array}{l}\text { Clinics of surgery } \\
\text { group }\end{array}$ & $\begin{array}{l}\text { Clinics of gynecology } \\
\text { group }\end{array}$ \\
\hline Domain & $\begin{array}{c}\text { Dimension of Approach } \\
\text { coefficient } 1\end{array}$ & 65.14 & 79.69 & 68.08 \\
\hline Education & Dimension of Deployment coefficient 2 & 127.8 & 133.27 & 130.54 \\
\hline Program & Dimension of Learning coefficient 3 & 184.98 & 185.15 & 187.16 \\
\hline Domain & Dimension of Integration coefficient 4 & 240.43 & 243.60 & 242.37 \\
\hline Total score (of 1000) & & 618 & 631 & 627 \\
\hline percentage & & $61.8 \%$ & $63.1 \%$ & $62.7 \%$ \\
\hline Result & & Appropriate & Appropriate & Appropriate \\
\hline
\end{tabular}

low score in the indicator of existence of evaluation system for practical and clinical education in colleagues' opinion (EESPCECO).

Internal medicine group of clinics' performance scored medium in this domain (Table 3). In this domain, the most appropriate index was CEEO, while TV and CA scored medium. In TV and CA indexes, there was considerable discrepancy between the score gained from surveying the documents and interviewing methods and observation method. This discrepancy was due to the difference in individual and organizational status of the participants and the effect this difference had on their attitudes. This means that both clear and fixed methods of evaluation and valid and stable tests in students' educational life are highly important; and with regards to cognitive dissonance theory (26), this importance leads to colossal influence of any unfavorable prior experience on framing their attitudes. On the other hand, it is quite natural for professors who, for years, have assessed their students with relatively stable evaluative methods and considered them as being completely clear, credible, and valid. It is suggested to those in charge to pay special attention to validation of these tests.

The performance of the gynecology group was medium in evaluation domain (Table 2). In this domain, the most appropriate indexes were PAEP, TV, and CEEO, which compared to standards of this study, gained medium scores, and the weakest index was CA that gained weak scores in all the 3 methods of data collection.

\section{The domain of training and research resources (TRR)}

The surgery group clinics showed weak performance in this domain (Table 3). The main weakness in this domain was due to weak performance in research specifications and facilities (RSF). In this domain, the most appropriate index was commensurability per capita and with standards (CPCS), and in the meantime, ambulatory education facilities and learning spaces (AEFLS) scored medium. Although the indicators of RFS index in surveying the documents method and interviewing method scored very weak, the experts considered medium scores in their observations for this indicator. Hence, it can be concluded that the ambulatory training learning space is potentially a suitable source for conducting research and that education officials and administrators should take the necessary measures to properly take advantage of this rich source.

The internal medicine group of clinics' performance scored medium in TRR domain (Table 3). In this domain, the most appropriate index was CPCS, and AEFLS and RFS were medium.

The performance of the gynecology group in TRR domain was medium (Table 3 ). In the domain, the most appropriate index was CPCS and the weakest was RSF, while AEFLS index scored medium.

Table 2. The status of evaluation domain based on national standards of ambulatory training in Firoozgar hospital using Baldrige Excellence Model Matrix

\begin{tabular}{|c|c|c|c|c|}
\hline & $\begin{array}{c}\text { Total score } \\
\text { Triangulation and weighting }\end{array}$ & $\begin{array}{l}\text { Clinics of internal } \\
\text { medicine group }\end{array}$ & $\begin{array}{l}\text { Clinics of surgery } \\
\text { group }\end{array}$ & $\begin{array}{l}\text { Clinics of gynecology } \\
\text { group }\end{array}$ \\
\hline & $\begin{array}{c}\text { Dimension of Approach } \\
\text { coefficient } 1\end{array}$ & 52.95 & 62.91 & 49.5 \\
\hline Evaluation & Dimension of Deployment coefficient 2 & 97.9 & 114.13 & 93.5 \\
\hline \multirow[t]{5}{*}{ Domain } & Dimension of Learning coefficient 3 & 142.8 & 167.75 & 137.12 \\
\hline & Dimension of Integration coefficient 4 & 188.15 & 219.33 & 178.5 \\
\hline & Total score (of 1000) & 481 & 563 & 458 \\
\hline & Percentage & $48.1 \%$ & $56.3 \%$ & $45.8 \%$ \\
\hline & Result & Medium & Medium & Medium \\
\hline
\end{tabular}

Table 3. The status of training and research resources domain based on national standards of ambulatory training in Firoozgar hospital using Baldrige Excellence Model Matrix

\begin{tabular}{|c|c|c|c|c|}
\hline & $\begin{array}{c}\text { Total score } \\
\text { Triangulation and weighting }\end{array}$ & $\begin{array}{l}\text { Clinics of internal } \\
\text { medicine group }\end{array}$ & $\begin{array}{l}\text { Clinics of surgery } \\
\text { group }\end{array}$ & $\begin{array}{l}\text { Clinics of gynecology } \\
\text { group }\end{array}$ \\
\hline \multirow{7}{*}{$\begin{array}{l}\text { Research and } \\
\text { education } \\
\text { domain }\end{array}$} & $\begin{array}{c}\text { Dimension of Approach } \\
\text { coefficient } 1\end{array}$ & 42.55 & 54.41 & 48.12 \\
\hline & Dimension of Deployment coefficient 2 & 82.5 & 106 & 96.12 \\
\hline & Dimension of Learning coefficient 3 & 116 & 157.08 & 142.25 \\
\hline & Dimension of Integration coefficient 4 & 156.3 & 203.97 & 187.37 \\
\hline & Total score (of 1000) & 397 & 521 & 473 \\
\hline & Percentage & $39.7 \%$ & $52.1 \%$ & $47.3 \%$ \\
\hline & Result & Weak & Medium & Medium \\
\hline
\end{tabular}


Table 4. The status of faculty members domain based on national standards of ambulatory training in Firoozgar hospital using Baldrige Excellence Model Matrixs

\begin{tabular}{|c|c|c|c|c|}
\hline & $\begin{array}{c}\text { Total score } \\
\text { Triangulation and weighting } \\
\end{array}$ & $\begin{array}{l}\text { Clinics of internal } \\
\text { medicine group }\end{array}$ & $\begin{array}{l}\text { Clinics of surgery } \\
\text { group }\end{array}$ & $\begin{array}{l}\text { Clinics of gynecology } \\
\text { group } \\
\end{array}$ \\
\hline \multirow{7}{*}{$\begin{array}{l}\text { Faculty members } \\
\text { domain }\end{array}$} & Dimension of Approach coefficient 1 & 67.26 & 71.81 & 62.49 \\
\hline & Dimension of Deployment coefficient 2 & 128.99 & 141.88 & 130.83 \\
\hline & Dimension of Learning coefficient 3 & 192.19 & 211.66 & 194.99 \\
\hline & Dimension of Integration coefficient 4 & 254.39 & 281.55 & 257.16 \\
\hline & Total score (of 1000) & 643 & 705 & 645 \\
\hline & Percentage & $64.3 \%$ & $70.5 \%$ & $64.5 \%$ \\
\hline & Result & Appropriate & Appropriate & Appropriate \\
\hline
\end{tabular}

\section{The domain of faculty members (FM)}

All 3 groups of the clinics had an appropriate performance in this domain (Table 4), which was due to appropriate performance of all 3 indexes of this domain in the 3 groups of clinics.

\section{Discussion}

\section{The domain of education programs (EP)}

All 3 groups of the clinics observed national standards in this domain at the appropriate level. Baldrige Scoring Matrix also showed a satisfactory status for the 3 groups. Medium performance scores in preparing and communicating educational objectives and professors and students' duties (PCEOPSD) subcategory in the clinics of surgery group pinpoints the fact that the educational program that is prepared in the format of a booklet titled, "study guide" is either not distributed properly among students or not taken as seriously as its significance mandates.

In the national standards of medical education, in EP domain, the emphasis is on the relevance of social and behavioral sciences with clinical activities. In Firoozgar hospital's education program, in compliance with realization of these objectives, there are somewhat planning and some endeavors, but it is hardly sufficient and there are also in-service trainings in the existing trainings of the program to empower the graduates with clinic management and pay due attention to people's physical, spiritual, mental, and social health.

Also, the opinion polls have found that generally there is no positive attitude towards communicational skills in the patient-physician relationship. Thus, to improve the status of this skill, necessary targeting and trainings should be done and doctors should learn those communicational skills and be obliged to observe them. Moreover, following topnotch methods and approaches of education and modern technology including bolstering IT and utilizing education's scientific principles can be helpful in compensating for the deficits of the program.

Farzianpur et al. study in Tehran University of Medical Sciences, which has been conducted using internal evaluation method based on interviewing managers and faculty members of 9 departments, revealed $73 \%$ conformity between EP domain of the university and the basic level of WFME guideline (9). This endorses the average results of EP domain in the present study $(62 \% / 64 \%)$. However, Mirzadeh et al. study conducted in Tehran University of Medical Sciences, performed using data collection, observation, and interviewing, and sending questionnaire to all people involved including professors, students, and managers, suggested that the status of medical education in Tehran University of Medical Sciences is medium or higher than national standards' recommendations, which themselves are derived from the basic level of WFME guideline, in only $40 \%$ of the cases (17).

Shekarchi et al. study in the AJA's Medical University, which was conducted using document review method, showed conformity of $54 / 37 \pm 9.03 \%$ between this university's EP and the basic level of WFME guideline (22), which is commensurate with our present study.

Khaje-Azad et al. study in Baghiat Allah University indicated that the quality of EP based on WFME guidelines is "between basic and quality" level according to professors and "lower than basic" level according to students and is appropriate based on the national standards according to the professors, and weak according to the students (11), which considering the national standards results, the professors' opinion conforms to the results of the present study.

Karimian et al. study in Shiraz University of Medical Sciences suggested that according to head of departments, EP domain in that university has had an appropriate performance in curriculum design index and utilizing new educational approaches (UNEA) index (12). The results of this study in detecting appropriate indexes conform to the findings of the present study in UNEA.

Ashurian et al. study in Isfahan University of Medical Sciences stated that EP domain in that university in medicine was $80 \%$ commensurable with national standards of medical education of Iran (7), which supports the results of the present study.

\section{The domain of evaluation}

In the evaluation domain, all 3 groups of clinics follow national standards in medium level. One of the strengths of the evaluation domain in Firoozgar hospital's ambulatory training is the existence of a prearranged process and program for assessing the students in all 3 groups of clinics and also in a guidebook titled, "Clinical Lesson Plan and Log Book". In the section related to "Recording the Information about Presence in the Hospital", the emphasis is on visiting the patient, how to record patient's history, performing careful examination, registering accurate and correct data, and treating the patient properly; in addition, at the beginning of each note book the objectives of each course are mentioned.

Even though there are various assessments including evaluating professors, students and tests, there is no satisfactory and practical mechanism to give feedback to those 
who are evaluated, do follow-ups, and monitor the assessments. Also, validity and reliability of the assessment tools are not verified.

Shekarchi et al. study in AJA's Medical University, which has been performed applying document review method, demonstrated conformity of $50 \pm 4 / 24 \%$ between evaluation domain in this university and the basic level of WFME guidelines (22), which is commensurate with the findings (48.06) of the present study. However, in Farzianpur et al. study in Tehran University of Medical Sciences, which has been conducted using internal evaluation method based on interviewing managers and faculty members of 9 departments, the percentage of conformity of evaluation domain of that university with the basic level of WFME guideline was 71.5 (9).

Khaje-Azad et al. study in Baghiat Allah University indicated that the quality of evaluation domain based on WFME guidelines is "between basic and quality" level and based on the national standards is appropriate (11), which confirms the results of the present study.

Ashurian et al. study in Isfahan University of Medical Sciences pinpoints that the evaluation domain of that university in medicine in $61 \%$ of the cases conforms to the national standards and is semi-appropriate (7), which verifies the findings of our study.

Karimian et al. study in Shiraz University of Medical Sciences reveals that more than $50 \%$ of the head of the departments in Medical Faculty considered VE index to be in an inappropriate statue (12), which substantiates the findings of our study in rendering this index as not appropriate.

\section{The domain of training and research resources (TRR)}

In this domain, surgery group of the clinics scored weak in observing the standards, but the other 2 scored somewhat medium. Lack of adequate facilities for pursuing and conducting research projects was the primary reason of poor performance in this domain. Although utilizing richly valuable potentials like research centers in clinics - especially education-based clinics - is an element that can be quite effective and helpful and noting the mechanisms, which have been taken into account in this field; namely, noticing research priorities in dissertations, students' active participation in research projects, establishing research centers for students in hospitals, sufficient supporting for exceptional talents' researches, and constructive and purposeful interactions with other credible scientific institutes. There is no clear definition of facilities and research activities suitable for ambulatory learning spaces; therefore, it is the responsibility of education officials of the hospitals to ratify policies for the hospital clinics that support research in education.

To this end, supporting the education development office of the hospital is of utmost importance.

Shekarchi et al. study in AJA's Medical University, which has been performed using document review method, demonstrated conformity of $45.83 \% \pm 14.28$ between the status of TRR domain of this university and the basic level of WFME guidelines in TRR domain (22), which corroborates the findings of our study in this domain $(46.37 \%)$.

Farzianpur et al. study in Tehran University of Medical
Sciences, which has been conducted using internal evaluation method based on interviewing managers and faculty members of 9 departments, suggested $62.2 \%$ conformity between TRR domain of this university and the basic level of WFME guideline (9), which endorses the results of the present study.

Ashurian et al. study in Isfahan University of Medical Sciences revealed that the TRR domain of that university in medicine in $54 \%$ of the cases conforms to the national standards and is semi-appropriate (7), which substantiates the findings of our study.

\section{The domain of faculty members (FM)}

In FM domain, all the 3 groups of clinics observed the national standards of undergraduate medical education in an appropriate level, but they should endeavor to eradicate such minor defects such as the absence of a plan to develop faculty members, the disproportionateness of faculty members number and activities to their various roles including research, scientific development, promoting community's health, streamlining, and guiding exceptional talents, and providing professional services, which all resulted from defects in planning and guiding specialized and skillful manpower to enable the faculty members to optimally spend all their energy and time on teaching, researching, taking care of the patients, and managing their responsibilities.

Shekarchi et al. study in AJA's Medical University, which has been conducted using document review method, suggested conformity of $65 \% \pm 7.07$ between the status of FM domain of this university and the basic level of WFME guidelines in FM domain (22), which validates the findings of our study in this domain $(68.98 \%)$.

Farzianpur et al. study in Tehran University of Medical Sciences, which has been conducted using internal evaluation method based on interviewing managers and faculty members of 9 departments, revealed $83.8 \%$ conformity between FM domain of this university and the basic level of WFME guideline, (9) in which, similar to our study, the staff were not studied and only the indexes about the faculty members were checked, and likewise the performance was appropriate.

Ashurian et al. study in Isfahan University of Medical Sciences revealed that the FM domain of that university in medicine in $74 \%$ of the cases conforms to the national standards and is appropriate (7), which verifies the findings of our study.

Khaje-Azad et al. study in Baghiat Allah University indicated that the quality of FM domain based on WFME guidelines is "between basic and quality" level and based on the national standards, is appropriate according to the professors and is weak according to students (11), which considering professors' opinions in the study based on the national standards approves the results our study.

\section{Conclusion}

Two domains of EP and FM were the most successful domains being studied in Firoozgar hospital, based on the national standards of undergraduate medical education in Iran, and the other 2, validation domain and TRR, had a medium performance. 
According to these findings, ambulatory training in Firoozgar hospital, based on the national standards of undergraduate medical education in Iran, in 4 domains of education program, validation, training and research resources, and faculty members was evaluated to be average. Therefore, it is suggested that considering the national standards of undergraduate medical education, ambulatory training should be provided and implemented in Firoozgar hospital and the necessary measures should be taken to make revisions in domains and indexes, which in this study, it was found that they need some improvement.

Overall, according to Baldrige Matrix, there is an appropriate approach towards applying the national standards of ambulatory training in undergraduate medical education in Firoozgar hospital and most of the indexes have been deployed. In learning dimension, the main challenge was not using the systematic feedback of deploying guideline indexes' results in improving the quality of ambulatory training system. In integration dimension, the main challenge was the absence of a holistic view of the national standards of ambulatory training in undergraduate medical education and separate deployment of indexes in different domains of the standard. Since one of the most important sections of an evaluation research is offering suggestions to improve the plan, with regards to the results of this study, the following suggestions are put forward to improve the status of the 4 domains:

\section{In education program domain}

1. Using motivational strategies to further involve the faculty members in education programs related to ambulatory training

2. Paying more attention to professional codes of conduct via merging this category into clinical courses

3. Enforcing community-oriented education of general practitioners

4. Paying more attention to teaching ambulatory care by professors and providing them with new training strategies

5. Paying special attention to teaching appropriate diagnosis and prescription in ambulatory care

6. Paying attention to facilitating independent involvement of learners in clinics under the supervision of professors

\section{In validation domain}

1. Conducting supplementary ways of evaluating professors such as evaluating by partners

2 . Providing necessary mechanisms to present the professors with the feedback of evaluation results and reinforcing them

3. Placing more emphasis on education-oriented education in periodic evaluations of faculty members

\section{In training and research resources domain}

1. Using existing educational spaces and resources in the society, such as private clinics, to conduct research and education projects

2. Conducting research projects and evaluation programs to more precisely examine the status of education and research resources in ambulatory care training centers
3. Paying more attention to ambulatory training in programs supporting research projects of professors

4. Paying attention to improving learning spaces and expanding academic resources in clinics

\section{In faculty members domain}

1. Paying more attention to education management and leadership to have education planning, supervising, and objective evaluation of programs

2. Paying more attention to development of faculty members and consequently the development of scientific ability of the hospital and university

\section{Suggestions for further studies}

To evaluate ambulatory training status, future studies can involve more groups, such as graduates, graduate supervisors in health care system, patients referring to hospital clinics, and hospital staff, rather than just students, professors, and education managers.

\section{Conflict of Interests}

The authors declare that they have no competing interests.

\section{References}

1. Amini A, Alizadeh M, Farzaneh F. Having a check on the condition of ambulatory care education from the view of interns in therapeutic training sections of Tabriz. Iranian J Train Med Sci. 2002;7:19-21.

2. Azizi F. Medical sciences training: challenges and views. Tehran: Hygiene, Treat Med Train. 2003, Vol. 1.

3. Alizadeh M, Sadeghi HasanAbadi A, Tabatabaee HR, Sharifi B. A survey of employed general practitioners in therapeutic sanitary centers Booklet of summarized articles of $11^{\text {th }}$ geographical international conference and $1^{\text {st }}$ ambulatory care conference of Iran. Shiraz University of Medical Sciences: 116.

4. Bazargan A. Internal and collegiate assessing and its function in continuous improving of quality of higher education. Planning and research quarterly journal in higher education. 1995;4:49-69

5. Catton PA, Tallett SE, Sugar L, Rothman AI: A guide to internal program evaluation for Program directors. Annals RCPSC 1999; 32(8):441-447

6. Christensen L. The Bologna Process and medical education. Med Teach. 2004; 26 (7): 625-629.

7. Dehghani poode M, Shams B, Ashooriyon V, Esmaeeli A, Asiliyan A, Nasri P, et al. Internal assessment of program of general medical science of Esfahan based on basic standards of ministry of health, treatment and medical training. Providing a model for performing assessment and analysis of results. Iranian J Train Med Sci. 2010;5(10)5:552565 .

8. FarziyanPoor F, Accreditation. Ministry of health, treatment and medical training. Secretariat of supervision, assessment and spreading universities of medical sciences. Tehran University of Medical Sciences. 1999:5.

9. FarziyanPoor F, Emami AH, Kavoosi Z. Accreditation of Tehran University of Medical sciences by using WFME standards. Tehran: Ministry of Health, Treatment and Medical Education. 2008:213-214

10. Festinger L. A theory of cognitive dissonance, Stanford University Press. 1962:15.

11. KhajeAzad M, Zaraee A. Assessment and comparison of the quality of general medical training of Baghiyatalah University based on national and universal standards. Iranian $J$ Train Med Sci. 2010;429(4):410-417.

12. Karimiyan Z, Kajoori J, BazrAfkan L, Lotfi F, Amini M. Checking the attribute of management of medical training groups related to quality of education program and course planning based on national and universal standards. $10^{\text {th }}$ national Congress of Medical Training; 1388 , $15^{\text {th }}$ to $17^{\text {th }}$ of Ordibehesht; Shiraz, Iran; 2009:43.

13. Mortazavi A, RazmAra A. Having a check on satisfaction of trainees 
and clinical interns of medicine from training section, emergencies, ambulatory centers within hospital and ambulatory centers within society in Esfahan University of Medical Sciences. Iranian journal of medical training in medical sciences. $5^{\text {th }}$ year; 14:45-52.

14. Mahvari Kh, Sadeghi HasanAbadi A, Tabatabaee HR. Making a check on clinical faculty members of Shiraz University of Medical Sciences for participation of doctors of private section in order to ambulatory care education to medicine students. Booklet of summarized articles of $11^{\text {th }}$ geographical international conference and $1^{\text {st }}$ ambulatory care conference of Iran. Shiraz University of Medical Sciences: 209.

15. Mohammadi R, FathAbadi J, YadgarZadeh Q, MirzaMohammadi M, Parand K. Assessing quality in higher education, concepts, principles, methods and criteria. Tehran Edu J. 2005;12-13.

16. Ministry of health, treatment and medical training of Islamic Republic of Iran. Secretariat of council of general medical sciences training. Basic standards for general medical sciences training in $15^{\text {th }} \mathrm{Mehr}$, 1388. Available at: http://scume.behdasht.gov.ir/uploads/172_277_ New-standard.pdf.

17. Mirzazade A, Moslehi H, Emami Razavi H, AzarPara A. The comparison of the condition of general medical training in medical college of Tehran University of Medical Sciences with national standards. Summarized articles of international congress of performing international standards of universal federation of medical training in general medical training program. Public relations of education vice chancellery. 2009:167.

18. Maccarrick G. A practical guide to using the World Federation for Medical Education Standards. WFME 2: educational program. Ir J Med Sci. 2010 Dec;179(4):489-491.

19. Peyvandi A, Nazari A, Maddah Sh. The study of comments of employed faculty members and medicine students of university of medical sciences and hygienic services of Semnan related to ambulatory care education. Booklet of summarized articles of $11^{\text {th }}$ geographical international conference and $1^{\text {st }}$ ambulatory care conference of Iran. Shiraz University of Medical Sciences: 84.

20 Tousignant B, Du Toit R. Adapting the World Federation for Medical Education standards for use in a self-audit of an eye care training programme. Educ Health (Abingdon). 2011 Dec; 24(3): 641.21. Pulido MPA, Cravioto A, Pereda A, Rondon R, Pereira G, et al. Changes, trends and challenges of medical education in Latin America. Med Teach. 2006;28(1):24-29.

21.Shaygah B, Ahmadi A. Conducting a survey of medicine students related to the quality of plans of community based ambulatory education in therapeutic sanitary and education centers of shahid navvab safavi of Esfahan in 1375. Booklet of summarized articles of $11^{\text {th }}$ geographical international conference and $1^{\text {st }}$ ambulatory care conference of Iran. Shiraz University of Medical Sciences: 37.

22. Shekarchi B, FarokhNejad Z, Rafiee M, et al. Assessing general medical training in university of medical sciences of Army of Islamic republic of Iran with the use of WFME standards in the education domain. Tehran: Ministry of Health, Treatment and Medical Education. 2008:223-224.

23. Simunovic VJ, Sonntag HG, Hren D, Dorup J, Krivokuća Z, Bokonjic $\mathrm{D}$, et al. A comprehensive assessment of medical schools in Bosnia and Herzegovina. Med Educ. 2006Dec;40(12):162-172.

24. YarMohammadian MH, Kalbasi A. Internal assessing of education groups of management and medical notices collage of Esfahan University of Medical sciences. Iranian J Train Med Sci. 2006;6(1):125-137.

25. Yamani Doozi Sorkhaee M. An introduction to the review of collegiate systems. Tehran: Shahid Beheshti University publication. 2001

26. Shahabudin SH. Medical education today: globalising with quality. Med J Malaysia. 2005;60:4. 